\title{
Ultrasound and Technetium-99m Sestamibi Scintigraphy Diagnostic Performance as Preferred Localization Techniques in Patients with Primary Hyperparathyroidism: A Literature Review
}

\author{
Diani Kartini ${ }^{1}$, Sonar S Panigoro ${ }^{2}$, Ciputra Linardy ${ }^{3}$
}

\section{ABSTRACT}

Introduction: Primary hyperparathyroidism (PHPT) is a medical problem whose definitive management is surgery. Preoperative imaging studies for identifying the solitary parathyroid adenoma are a requirement for focused parathyroidectomy in patients with PHPT. There are various imaging modalities for the localization study. Nevertheless, up until now, the gold standard for parathyroid imaging has still not been established. Accurate parathyroid imaging is needed in PHPT cases to support the success of focused parathyroidectomy and prevent increasing patient morbidity due to bilateral neck exploration.

Aims and objectives: It is very important for this study to investigate the positive predictive value (PPV) and sensitivity of sestamibi and ultrasonography as preoperative localization techniques.

Materials and methods: Literature search has been carried out on Cochrane, PubMed, and the ScienceDirect database site, using a combination of Medical Subject Headings (MeSH) search terms and keywords: "parathyroidectomy," "primary hyperparathyroidism," "parathyroid adenoma," "ultrasound,"' ultrasonography,"'radionuclide imaging,"'sestamibi,"'sensitivity and specificity," and "predictive values of tests." was conducted to look for previous publications, about the sensitivity and positive predictive value of ultrasonography as a technique of localizing preoperative compared with sestamibi in localizing parathyroid adenomas in patients with PHPT. After research articles have been obtained, a selection and examination of journals according to the inclusion and exclusion criteria was conducted.

Results: A total of 192 studies were obtained, which are Cochrane (0) study, PubMed (33) studies, and ScienceDirect (159) studies. After screening, 15 studies are selected for review. According to the studies, ultrasonography has a sensitivity of 55-100\% and PPV 74-100\%. Ultrasonography has a wide range of sensitivity because it is highly operator-dependent depending the expertise of the operator. Ultrasonography by radiologists has a sensitivity of $55-94.6 \%$ and PPV 74-97.2\%, while ultrasonography by a surgical specialist has a sensitivity of $83-93.2 \%$ and PPV $80-85.1 \%$. Sestamibi has a sensitivity of $64-93.3 \%$ and PPV $70.5-100 \%$. Limitation of sestamibi includes poor spatial resolution causing low precision in anatomic localization, and there is the use of ionizing radiation. While SPECT/CT has similar sensitivity in localizing parathyroid adenomas located on the retrothyroid and ectopic sites (sensitivity $86.7 \%$ and $81.5 \%$; PPV $98.1 \%$ and $100 \%$ ). The study has found that adding ultrasonography to the SPECT/CT examination increased sensitivity, but decreased PPV.

Conclusion: Ultrasound examination is very dependent on the operator so that sensitivity varies, influenced by the expertise and experience of the operator in each institution. Ultrasonography can be used as the main modality in localization studies in patients with PHPT in areas that do not have nuclear radiology facilities.

Keywords: Parathyroidectomy, Primary hyperparathyroidism, Radionuclide imaging, Sestamibi, Ultrasonography. World Journal of Endocrine Surgery (2020): 10.5005/jp-journals-10002-1284
\end{abstract}

\section{INTRODUCTION}

Primary hyperparathyroidism (PHPT) is a medical problem whose definitive management is surgery. ${ }^{1-3}$ Preoperative imaging studies for identifying the solitary parathyroid adenoma are a requirement for focused parathyroidectomy in patients with PHPT. ${ }^{3}$ However, there is no universally accepted algorithm for imaging the parathyroid gland, and the choice of imaging is influenced by the preferences of the surgeons. Previous studies have yielded varying sensitivities of parathyroid imaging modalities., ${ }^{4,5}$

Both ultrasonography and sestamibi have advantages and disadvantages. Noninvasive, rapid ultrasonography can evaluate lesions in the thyroid gland, but there are limitations in evaluating mediastinal parathyroid glands. ${ }^{4,6,7}$ Sestamibi can detect ectopic and posterior glands that are not detected on ultrasonographic examination, but the radiation used can increase cancer risk. ${ }^{4}$ Besides, there are only 17 hospitals in Indonesia that have nuclear radiology facilities. ${ }^{8}$
${ }^{1-3}$ Department of Surgery, Cipto Mangunkusumo Hospital, Universitas Indonesia, Jakarta, Indonesia

Corresponding Author: Ciputra Linardy, Department of Surgery, Cipto Mangunkusumo Hospital, Universitas Indonesia, Jakarta, Indonesia, Phone: +628158709821, e-mail: d.kartini@gmail.com, linardy.c@gmail. com

How to cite this article: Kartini D, Panigoro SS, Linardy C. Ultrasound and Technetium-99m Sestamibi Scintigraphy Diagnostic Performance as Preferred Localization Techniques in Patients with Primary Hyperparathyroidism: A Literature Review. World J Endoc Surg 2020;12(1):9-13.

Source of support: Nil

Conflict of interest: None

Until now there has been no standard or algorithm for parathyroid imaging. Ultrasonography is a publicly available

(c) The Author(s). 2020 Open Access This article is distributed under the terms of the Creative Commons Attribution 4.0 International License (https://creativecommons. org/licenses/by-nc/4.0/), which permits unrestricted use, distribution, and non-commercial reproduction in any medium, provided you give appropriate credit to the original author(s) and the source, provide a link to the Creative Commons license, and indicate if changes were made. The Creative Commons Public Domain Dedication waiver (http://creativecommons.org/publicdomain/zero/1.0/) applies to the data made available in this article, unless otherwise stated. 
modality, both in urban and rural areas, while scintigraphy is only available in certain hospitals. Accurate parathyroid imaging is needed in PHPT cases to support the success of focused parathyroidectomy and prevent increasing patient morbidity due to bilateral neck exploration. Therefore, it is necessary to study the sensitivity and PPV of ultrasonography and sestamibi as preoperative localization techniques.

\section{Aims and Objectives}

To determine the sensitivity and positive predictive value (PPV) of ultrasonography and sestamibi preoperative localization techniques for localizing parathyroid adenomas in patients with PHPT.

\section{Materials and Methods}

A literature search was carried out to look for previous publications, which raised research questions about the sensitivity and positive predictive value of ultrasonography as a technique of localizing preoperative compared with sestamibi in localizing parathyroid adenomas among patients with PHPT. The study was conducted at Division of Surgical Oncology, Department of Surgery, Cipto Mangunkusumo Hospital, in December 2018February 2019. After the research question ("how is ultrasound sensitivity and PPV as preoperative localization techniques compared with sestamibi in localizing parathyroid adenomas in patients with PHPT?") and the person, intervention, comparison, outcome (PICO) components were identified, a search and selection of literature on Cochrane, PubMed, and Science Direct database was conducted. This was done by using a combination of Medical Subject Headings (MeSH) search terms and keywords: "parathyroidectomy," "primary hyperparathyroidism," "parathyroid adenoma," "ultrasound," "ultrasonography," "radionuclide imaging," "sestamibi," "sensitivity and specificity," and "predictive values of tests." After research articles from each database have been obtained, a selection and examination of journals according to the inclusion and exclusion criteria was conducted to eliminate article duplication.

\section{Inclusion Criteria}

- The study is in the form of meta-analysis, systematic review, randomized controlled trial, case series, and case report.

- In English language.

- Publication within the last 5 years.

\section{Exclusion Criteria}

- Publication in the form of correspondence, editorial, and commentary.

- Not available in the full-text format.

\section{Result and Discussion}

From the literature review, a total of 192 studies were obtained, which are Cochrane (0) study, PubMed (33) studies, and ScienceDirect (159) studies. After selecting the studies based on the inclusion and exclusion criteria, 10 studies from PubMed and 6 studies from ScienceDirect were selected. After that, the reviewer conducted a duplicate studies screening, which resulted in one study that was similar from both PubMed and ScienceDirect, resulting in
15 studies were selected for review. All literature is available in the full-text format; literature downloads are carried out, and assessment is carried out. The summary of the diagnostic performance on ultrasonography and sestamibi based on the literatures reviewed can be seen in Tables 1 to 6 .

Based on studies obtained in the literature review, ultrasonography has a sensitivity of $55-100 \%$ and PPV 74-100\%. Ultrasonography has a wide range of sensitivity in the 13 studied literature. Ultrasonography is highly operator-dependent, so the sensitivity varies, influenced by the expertise and experience of the operator in each institution. The lowest sensitivity, PPV, and ultrasound accuracy $(55,74$, and $48 \%$ ) were obtained in the study of Ryan et al. (2017), where ultrasonographic examinations were carried out in four radiology departments by different radiology specialists. This results in significant interexaminer variability and explains the low sensitivity in this study. ${ }^{9}$

Al-Kurd et al. (2018) state that the ultrasound performed by an experienced surgeon is superior to other modalities in determining the correct adenoma lateralization, and has the highest sensitivity and accuracy (93.2\% and $80.1 \%$ ) compared to other inspection ( $p<0.001$ ). The superiority of ultrasonography by experienced surgical specialists compared to ultrasonography by radiology specialists who may be inexperienced with PHPT patients can be due to several factors. First, the surgical specialists generally accept patients after ultrasound examination of radiology specialists and sestamibi were made, which leads to bias due to these results. In addition, ultrasound performed by a surgical specialist generally focuses only on the parathyroid and thyroid glands, while radiologists typically examine the entire structure of the neck soft tissue. Finally, surgeons are generally more willing to take risks and regard a borderline-looking structure as an adenoma, whereas radiology specialists are reluctant to include uncertainty in official radiology reports. ${ }^{10}$

The study of Uslukaya et al. shows that ultrasonography performed in a team consisting of surgical specialists and radiology specialists can produce sensitivity, PPV, and accuracy up to $100 \%$. However, the drawback of this study is the small sample size (30 people). ${ }^{11}$

Of the 13 literatures that included ultrasonography as one of the modalities of localization of surgery, 7 are done by radiologists, 1 is done by endocrine surgeons, 1 is done by radiologists and surgeons, 1 is done by collaboration between radiologists and surgeons, 1 is done by trained ultrasonographers, while 2 literatures do not mention specifically the operator of the ultrasound. Ultrasonography by radiologists has a sensitivity of 55-94.6\% and PPV 74-97.2\%, while ultrasonography by a surgical specialist has a sensitivity of $83-93.2 \%$ and PPV $80-85.1 \%$. Ultrasonography performed by trained ultrasonographers produces low sensitivity (58\%). Therefore, ultrasound sensitivity and PPV are influenced by the expertise of the operator.

Sestamibi has a sensitivity of $64-93.3 \%$ and PPV 70.5$100 \%$. Limitation of sestamibi includes poor spatial resolution causing low precision in anatomic localization, and there is the use of ionizing radiation. In addition, the presence of benign thyroid nodules can reduce sestamibi sensitivity and follicular cell thyroid neoplasms can cause false accumulation of sestamibi. ${ }^{12}$

In this study, 10 literatures analyzed sestamibi as a localization study of parathyroidectomy. In the literature with a sample size $<100$ people, sensitivity (85-93.3\%) and PPV (91-100\%) sestamibi are relatively high. However, in the literature with a sample size of 
Table 1: Sensitivity, specificity, predictive value, and ultrasound accuracy ${ }^{9-21}$

\begin{tabular}{|c|c|c|c|c|c|c|}
\hline Author, year & $N$ & Sensitivity (\%) & Specificity (\%) & PPV (\%) & NPV (\%) & Accuracy (\%) \\
\hline Scattergood et al., 2018 & 184 & 70 & 57 & 92 & 19 & 68 \\
\hline \multicolumn{7}{|l|}{ Al-Kurd et al., 2018} \\
\hline - Radiology & 397 & 70.5 & & 84.6 & & 62.5 \\
\hline - Surgery & 221 & 93.2 & & 85.1 & & 80.1 \\
\hline Argirò et al., 2018 & 46 & 89.1 & 97.5 & 93.2 & 95.6 & \\
\hline Ryan et al., 2017 & 129 & 55 & & 74 & & 48 \\
\hline \multicolumn{7}{|l|}{ Frank et al., 2017} \\
\hline - $3 \mathrm{D}$ & 52 & $84-92$ & & & & \\
\hline - $2 \mathrm{D}$ & 52 & $69-71$ & & & & \\
\hline Keutgen et al., 2016 & 24 & 100 & & 92 & & \\
\hline Coelho et al., 2016 & 55 & & & 89 & & \\
\hline Seyednejad et al., 2016 & 24 & 58 & & 100 & & 58 \\
\hline Medas et al., 2016 & 212 & 62.4 & & 92.6 & & \\
\hline Ibrahim and Elsadawy, 2015 & 40 & 94.6 & 66.6 & 97.2 & & 92.5 \\
\hline Uslukaya et al., 2015 & 30 & 100 & & 100 & & 100 \\
\hline Kluijfhout et al., 2015 & 54 & 63.2 & & 78.3 & & \\
\hline Hughes et al., 2014 & 1644 & 83 & & 80 & & \\
\hline
\end{tabular}

Table 2: Sensitivity, specificity, predictive value, and accuracy of sestamibi ${ }^{12-16,18-22}$

\begin{tabular}{|c|c|c|c|c|c|c|}
\hline Author, year & $N$ & Sensitivity (\%) & Specificity (\%) & PPV (\%) & NPV (\%) & Accuracy (\%) \\
\hline Scattergood et al., 2018 & 184 & 64 & 57 & 92 & 17 & 64 \\
\hline Al-Kurd et al., 2018 & 376 & 83.3 & & 70.5 & & 70.5 \\
\hline Argirò et al., 2018 & 46 & 83.6 & 98.3 & 95 & 93.7 & \\
\hline Frank et al., 2017 & 52 & 90 & & & & \\
\hline Keutgen et al., 2016 & 28 & 85 & & 96 & & \\
\hline Coelho et al., 2016 & 47 & & & 91 & & \\
\hline Medas et al., 2016 & 180 & 78.9 & & 89.9 & & \\
\hline Ibrahim and Elsadawy, 2015 & 40 & 89.5 & 100 & 100 & & 90 \\
\hline Uslukaya et al., 2015 & 30 & 93.3 & & 100 & & 93.3 \\
\hline Hughes et al., 2014 & 1165 & 66 & & 88 & & \\
\hline
\end{tabular}

Table 3: Sensitivity, specificity, predictive value, and accuracy of the combination of ultrasonography and sestamibi ${ }^{12-14,19,21}$

\begin{tabular}{|c|c|c|c|c|c|c|}
\hline Author, year & $n$ & Sensitivity (\%) & Specificity (\%) & PPV (\%) & NPV (\%) & Accuracy (\%) \\
\hline Scattergood et al., 2018 & 184 & 81 & 71 & 95 & 33 & 80 \\
\hline Argirò et al., 2018 & 46 & 93.4 & 98.3 & 95 & 98.3 & \\
\hline \multicolumn{7}{|l|}{ Frank et al., 2017} \\
\hline - $3 \mathrm{D}$ & 52 & 100 & & & & \\
\hline - $2 \mathrm{D}$ & 52 & 96 & & & & \\
\hline Ibrahim and Elsadawy, 2015 & 40 & 97.3 & 100 & 100 & & 97.5 \\
\hline Hughes et al., 2014 & 920 & & & 92 & & \\
\hline
\end{tabular}

Table 4: SPECT sensitivity, specificity, predictive value, and accuracy ${ }^{22}$

\begin{tabular}{|c|c|c|c|c|c|c|}
\hline Author, year & $n$ & Sensitivity (\%) & Specificity (\%) & PPV (\%) & NPV (\%) & Accuracy (\%) \\
\hline \multicolumn{7}{|l|}{ McCoy et al., 2018} \\
\hline - Solitary gland & 539 & 91 & & 85 & & 77 \\
\hline - Multiglandular & 94 & 49 & & 37 & & 22 \\
\hline
\end{tabular}

$\geq 100$ people, sensitivity (64-83.3\%) and PPV (70.5-92\%) sestamibi decreased.

The Scattergood et al. (2018) study found that the sensitivity of the combination of ultrasonography and sestamibi was higher compared to ultrasonography alone ( $81 \%$ and $70 \%) .{ }^{13}$ Likewise with the research of Frank et al. (2017), the sensitivity of a combination of sestamibi and ultrasonography (96-100\%) is higher than ultrasonography (69-92\%) or sestamibi alone (90\%). ${ }^{14}$ 
Ultrasound and Technetium-99m Sestamibi Scintigraphy Diagnostic Performance as Preferred Localization Techniques

Table 5: SPECT/CT sensitivity, specificity, predictive value, and accuracy $9,11,17,22,23$

\begin{tabular}{|c|c|c|c|c|c|c|}
\hline Author, year & $n$ & Sensitivity (\%) & Specificity (\%) & PPV (\%) & NPV (\%) & Accuracy (\%) \\
\hline \multicolumn{7}{|l|}{ McCoy et al., 2018} \\
\hline Solitary gland & 647 & 96 & & 90 & & 83 \\
\hline Multiglandular & 108 & 68 & & 53 & & 36 \\
\hline Ryan et al., 2017 & 161 & 73 & & 87 & & 67 \\
\hline Seyednejad et al., 2016 & 24 & 90 & & 90 & & 75 \\
\hline Koberstein et al., 2016 & 88 & 85.1 & & 98.7 & & 84.1 \\
\hline Retrothyroid & 88 & 86.7 & 96.4 & 98.1 & 77.1 & 89.8 \\
\hline Ectopic & 88 & 81.5 & 100 & 100 & 92.4 & 94.3 \\
\hline Kluijfhout et al., 2015 & 63 & 80.3 & & 93 & & \\
\hline
\end{tabular}

Table 6: Sensitivity, specificity, predictive value, and accuracy of the combination of USG and SPECT/CT'11

\begin{tabular}{|c|c|c|c|c|c|c|}
\hline Author, year & $n$ & Sensitivity (\%) & Specificity (\%) & PPV (\%) & NPV (\%) & Accuracy (\%) \\
\hline Ryan et al., 2017 & 119 & 66 & & 46 & & 38 \\
\hline Kluijfhout et al., 2015 & 54 & 84.2 & & 77.4 & & \\
\hline
\end{tabular}

Double-phase parathyroid imaging using $99 \mathrm{~m}$ Tc-MIBI and SPECT/CT is one of the reliable and accurate methods for assessing parathyroid adenoma surgery. This examination is better for preoperative identification and localization if compared to ultrasonography, $\mathrm{CT}$, and $\mathrm{MRI}^{23}$

The Koberstein et al. (2016) study found that localization of parathyroid adenomas located on the retrothyroid and ectopic sites had similar sensitivity and PPV using SPECT/CT (sensitivity $86.7 \%$ and $81.5 \%$; PPV $98.1 \%$ and $100 \%$ ). In addition, the probability of precise localization increases with increasing serum $\mathrm{PTH} .{ }^{23}$

McCoy et al. (2018) concluded that SPECT/CT provides a more reliable operating guide than SPECT alone. Although the two imaging techniques are not adequate predictors for multiglandular disease, SPECT/CT is $60 \%$ more accurate $36 \%$ and $22 \%$, with higher sensitivity and PPV compared to SPECT alone in identifying multiglandular disease (68\% and $49 \%$ sensitivity; PPV 53\% and 37\%). ${ }^{22}$ Ryan et al. (2017) stated that ultrasound and SPECT/CT are only $37 \%$ suitable in localizing adenomas. ${ }^{22}$ Kluijfhout et al. (2015) found that adding ultrasonography to the SPECT/CT examination increased sensitivity, but decreased PPV. ${ }^{11}$

Ultrasonography, if performed by a trained operator, can produce an equivalent or higher sensitivity and PPV compared to sestamibi so that it can be used as the main modality in localization studies in patients with PHPT. This can especially be applied in areas that do not have nuclear radiology facilities.

\section{Conclusion}

- Ultrasonography has a sensitivity of $55-100 \%$ and PPV of 74-100\%.

- Sestamibi has a sensitivity of $64-93.3 \%$ and PPV of $70.5-100 \%$.

- Ultrasound examination is very dependent on the operator so that sensitivity varies, influenced by the expertise and experience of the operator in each institution.

- Ultrasonography can be used as the main modality in localization studies in patients with PHPT in areas that do not have nuclear radiology facilities.

\section{References}

1. Pallan S, Khan A. Primary hyperparathyroidism: update on presentation, diagnosis, and management in primary care. Can Fam Physician 2011;57(2):184-189.

2. Tay Y-KD, Yeh R, Kuo JH, et al. Pre-operative localization of abnormal parathyroid tissue by $99 \mathrm{mTc}$-sestamibi in primary hyperparathyroidism using four-quadrant site analysis: an evaluation of the predictive value of vitamin D deficiency. Endocrine 2018;60(1):36-45. DOI: 10.1007/s12020-018-1528-1.

3. Wilhelm SM, Wang TS, Ruan DT, et al. The American association of endocrine surgeons guidelines for definitive management of primary hyperparathyroidism. JAMA Surg 2016;151(10):959-968. DOI: 10.1001/ jamasurg.2016.2310.

4. Kuzminski SJ, Sosa JA, Hoang JK. Update in parathyroid imaging. Magn Reson Imaging Clin N Am 2018;26(1):151-166. DOI: 10.1016/ j.mric.2017.08.009.

5. Mihai R, Simon D, Hellman P. Imaging for primary hyperparathyroidism-an evidence-based analysis. Langenbeck's Arch Surg 2009;394(5):765-784. DOI: 10.1007/s00423-0090534-4.

6. Cheung K, Wang TS, Farrokhyar F, et al. A meta-analysis of preoperative localization techniques for patients with primary hyperparathyroidism. Ann Surg Oncol 2012(2):577-583. DOI: 10.1245/ s10434-011-1870-5.

7. Udelsman R, Åkerström G, Biagini C, et al. The surgical management of asymptomatic primary hyperparathyroidism: proceedings of the fourth international workshop. J Clin Endocrinol Metab 2014;99(10):3595-3606. DOI: 10.1210/jc.2014-2000.

8. Syarifah F, Daftar Rumah Sakit yang Gunakan Teknologi Nuklir untuk Kanker. https://www.liputan6.com/health/read/2453125/ daftar-rumah-sakit-yang-gunakan-teknologi-nuklir-untuk-kanker. Published March 7, 2016. Accessed January 15, 2019.

9. Ryan S, Courtney D, Moriariu J, et al. Surgical management of primary hyperparathyroidism. Eur Arch Oto-Rhino-Laryngol 2017;274(12):4225-4232. DOI: 10.1007/s00405-017-4776-4.

10. Al-Kurd A, Levit B, Assaly M, et al. Preoperative localization modalities in primary hyperparathyroidism: correlation with postoperative cure. Surgery 2018;164(1):130-136. DOI: 10.1016/j.surg.2018.02.016.

11. Kluijfhout WP, Borel IHM, Valk GD, et al. Enabling minimal invasive parathyroidectomy for patients with primary hyperparathyroidism using Tc-99m-sestamibi SPECT-CT, ultrasound and first results of 18F-fluorocholine PET-CT. Eur J Radiol 2015. 3-9. 
12. Argirò $R$, Diacinti $D$, Sacconi $B$, et al. Diagnostic accuracy of $3 T$ magnetic resonance imaging in the preoperative localisation of parathyroid adenomas: comparison with ultrasound and 99mTcsestamibi scans. Eur Radiol 2018(11). DOI: 10.1007/s00330-0185437-8.

13. Scattergood S, Marsden M, Kyrimi E, et al. Combined ultrasound and sestamibi scintigraphy provides accurate preoperative localisation for patients with primary hyperparathyroidism. Ann R Coll Surg Engl 2018. 1-6.

14. Frank SJ, Goldman-yassen AE, Koenigsberg T, et al. Sensitivity of 3-dimensional Sonography in preoperative evaluation of parathyroid hyperparathyroidism. J Ultrasound Med 2017;36(9):1897-1904. DOI: 10.1002/jum.14245.

15. Keutgen XM, Nilubol N, Agarwal S, et al. Reoperative surgery in patients with multiple endocrine neoplasia type 1 associated primary hyperparathyroidism. Ann Surg Oncol 2016;23(Suppl 5):701-707. DOI: 10.1245/s10434-016-5467-x.

16. Coelho MCA, Beuren AC, Lopes CB, et al. Role of imaging tests for preoperative location of pathologic parathyroid tissue in patients with primary hyperparathyroidism. Endocr Pr 2016;22(9):1062-1068. DOI: 10.4158/EP151137.OR.

17. Seyednejad N, Healy C, Tiwari P, et al. Dual-energy computed tomography: a promising novel preoperative localization study for treatment of primary hyperparathyroidism. Am J Surg 2016;211(5):839-845. DOI: 10.1016/j.amjsurg.2016.01.003.
18. Medas F, Erdas E, Longheu A, et al. Retrospective evaluation of the preand postoperative factors influencing the sensitivity of localization studies in primary hyperparathyroidism. Int J Surg 2016;25:82-87. DOI: 10.1016/j.ijsu.2015.11.045.

19. Ibrahim EAG, Elsadawy ME. Combined Tc-99m sesta MIBI scintigraphy and ultrasonography in preoperative detection and localization of parathyroid adenoma. Egypt J Radiol Nucl Med 2015;46(4):937-941. DOI: 10.1016/j.ejrnm.2015.07.006.

20. Uslukaya O, Gumus M, Tasdemir B, et al. Improvement of minimally invasive parathyroidectomy outcomes by real time ultrasonography performed by a surgeon and radiologist team. Med Ultrason 2015;17(3):315-321. DOI: 10.11152/mu.2013.2066.173.oky.

21. Hughes DT, Sorensen MJ, Miller BS, et al. The biochemical severity of primary hyperparathyroidism correlates with the localization accuracy of sestamibi and surgeon-performed ultrasound. J Am Coll Surg 2014;219(5):1010-1019. DOI: 10.1016/j.jamcollsurg.2014.06.020.

22. Mccoy KL, Ghodadra AG, Hiremath TG, et al. Sestamibi SPECT/ CT versus SPECT only for preoperative localization in primary hyperparathyroidism: a single institution 8-year analysis. Surg 2018;163(3):643-647. DOI: 10.1016/j.surg.2017.10.064.

23. Koberstein W, Fung C, Romaniuk K, et al. Accuracy of dual phase single-photon emission computed tomography/computed tomography in primary hyperparathyroidism: correlation with serum parathyroid hormone levels. Can Assoc Radiol J 2018;67(2):115-121. DOI: 10.1016/j.carj.2015.06.002. 